\title{
Naturalistic decision-making: continuous, open-world, and recursive
}

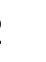
4

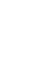
6 7

1 Department of Neuroscience, Center for Neuroengineering, Center for Magnetic Resonance Research, University of Minnesota, Minneapolis MN 55455

Seng Bum Michael Yoo ${ }^{1}$, Benjamin Yost Hayden¹, and John M. Pearson²

\section{2} Center for Cognitive Neuroscience, Department of Neurobiology, Department of Electrical and Computer Engineering Duke University, Durham, NC 27708

\section{Corresponding author:}

\section{Seng Bum Michael Yoo}

Department of Neuroscience and Center for Neuroengineering University of Minnesota, Minneapolis MN 55455

Email: sbyoo.ur.bcs@gmail.com

\section{Competing interests}

The authors have no competing interests to declare.

\section{Acknowledgements:}

This work is supported by an R01 from NIH (DA038615) to BYH. 


\section{ABSTRACT}

36 Real-world decisions take place over time, in dynamics contexts, and with

37 frequently shifting choice sets. In contrast, most studies of decision making have focused

38 on single, abstracted choices among small numbers of discrete options. Here, we advocate

39 for a shift in emphasis to what we call continuous decisions, which better capture the

40 complexity of decision-making in the wild. Continuous decisions involve a continuum of

41 possible responses and take place over an extended period of time during which the

42 response is continuously subject to modification. The range of options available at any

43 time can fluctuate and is affected by recent responses, making consideration of feedback

44 between choices and the environment essential. The study of continuous decisions

45 emphasizes distinct questions not easily captured by discrete decisions, including questions

46 about how the brain integrates choices with movement, bridges representations through

47 time, and maintains hierarchically organized information. While microeconomic theory has

48 proven invaluable for discrete decisions, we propose that engineering control theory may

49 serve as a better foundation for continuous ones. And while the concept of value has

50 proven foundational for discrete decisions, goal states and policies may prove more useful

51 for continuous ones.

52 


\section{Introduction}

Decisions as studied in the lab are typically discrete. Consider, for example, a

57 paradigmatic example, the Allais Paradox (Allais, 1953, Figure 1). It is one of the most influential artificial choices of the 20th century, and one that, probably more than any

59 other, inaugurated behavioral economics. The Allais Paradox involves a single choice

60 between a pair of categorically distinct, fully-specified options. It is the prototype of the

61 vast majority of decisions studied in microeconomics, behavioral economics, and

62 neuroeconomics (Kahneman and Tversky, 1979, Glimcher and Fehr, 2013). The decision-

63 maker has, in principle, as long as they want to decide. The result of the choice does not

64 affect what options are made available in the future. The choice itself takes place either

65 instantaneously or irrespective of time, and, once made, it is irrevocable. The decision-

66 maker can express their preference verbally, with a button press, or with any other

67 response modality. The decision-maker's choice is free of context; that is, it is disembodied

68 (Lepora and Pezzulo, 2015; Pezzulo and Cisek, 2016).

The Allais paradox is the epitome of what one might call a discrete decision. Many

70 of the decisions we make in our daily lives approximate such discrete decisions (Platt and

71 Huettel, 2008). For example, selecting an item from a menu in a restaurant and selecting a

72 life insurance plan can be conveniently thought of as discrete decisions. However, many

73 other decisions we face in our daily lives have different characteristics. Even quotidian

74 decisions may involve a continuum of options or a changing option set. They can take 
75 place over time and are enacted through complex movements (i.e. they are embodied).

76 They may take place with varying levels of conscious awareness and involve feedback

77 between the decider and the external world. Here we use the term continuous decisions to 78 describe them.

79 The major goal of the present article is to introduce and motivate the study of 80 continuous decisions. As we will argue, such decisions capture aspects of real behavior

81 often neglected in the fields that comprise decision science, but they offer potentially

82 unique insights into natural behavior. For concreteness, consider - in contrast to the Allai

83 paradox - a canonical continuous decision: the pursuit behavior of the window-skimmer

84 dragonfly (Libellula luctuosa). As it pursues its prey (a mosquito), the dragonfly must

85 continuously monitor the mosquito's position while both move rapidly through three-

86 dimensional space (Gonzalo-Bellido et al., 2016; Gonzalo-Bellido, 2020). Here, there is no

87 longer any obvious discrete decision among fixed options: rather, the dragonfly must

88 carefully regulate its wings to stabilize its body, track and extrapolate the future position of

89 the prey, and plot an intercept course. Actions at each moment come from a large

90 continuum of possibilities, each flowing into the next. Moreover, the mosquito will change

91 its behavior based on what the dragonfly does, and the dragonfly can exploit its knowledge

92 of that fact to improve its foraging.

93 While such complex behavior may be conceptualized or modeled mathematically

94 as a series of discrete economic choices, one at each moment, we argue that something in

95 this formulation fails to do the dragonfly justice. Because while the overall behavior

96 apparently involves a deliberate selection of flight plan and final strike time, as well as a 
97 balancing of costs (time, energy, risk) and benefits (caloric intake), it seems clearer to view

98 the notion of decision as encompassing the entire process, from the continuum of motor

99 adjustments to the selection of flight control strategies. From this perspective, the choice

100 first posed by Allais appears as a degenerate example, one in which the various aspects are

101 discretized, disembodied, and collapsed into a single moment. Even though it is a special

102 case, economics, as studied in psychology and neuroscience, has focused almost

103 exclusively on the discrete case and ignored the much larger world of continuous

104 decisions.

105 But considering decisions more broadly is valuable. From our perspective, the

106 greatest of these is the benefits to neuroscience that come from studying the types of

107 decisions our brains were evolved to handle (Brown and De Bivort, 2018; Calhoun and

108 Hayden, 2015; Kacelnik et al., 2011; Krakauer et al., 2017; Pearson et al., 2014). Indeed,

109 differences in sensory coding between artificial and natural stimuli are often stark (Brenner

110 et al., 2000; Vinje and Gallant, 2000; David et al., 2004), raising the possibility that

111 conclusions about choice coding derived from highly circumscribed and overtrained

112 paradigms may be less general than hoped. In addition, continuous decisions require

113 careful consideration of the sensorimotor context in which they are embedded. Rather than

114 attempt to divorce decision as cleanly as possible from perception and action, studying

115 continuous decisions requires us to confront the widespread observation that neural

116 representations often intermingle information about decisions, actions, and outcomes, even

117 in putative "decision" areas (Fusi et al;, 2013; Yuste, 2015 Fusi et al., 2016). Finally,

118 natural decisions focus on dynamics. Here again, while systems neuroscience has gained 
tremendous insights from the study of steady-state coding and ballistic movement, natural actions are primarily characterized by transition and the absence of equilibrium. Indeed, several of the most exciting recent ideas in decision-making have looked to decision dynamics as key (e.g. Mante et al., 2013; Resulaj et al., 2009; Stoke et al., 2013; Kobak et al., 2016).

\section{Option 1: \$1 Million}

Option 2: 1\% nothing $89 \% \$ 1$ Million $10 \% \$ 5$ Million

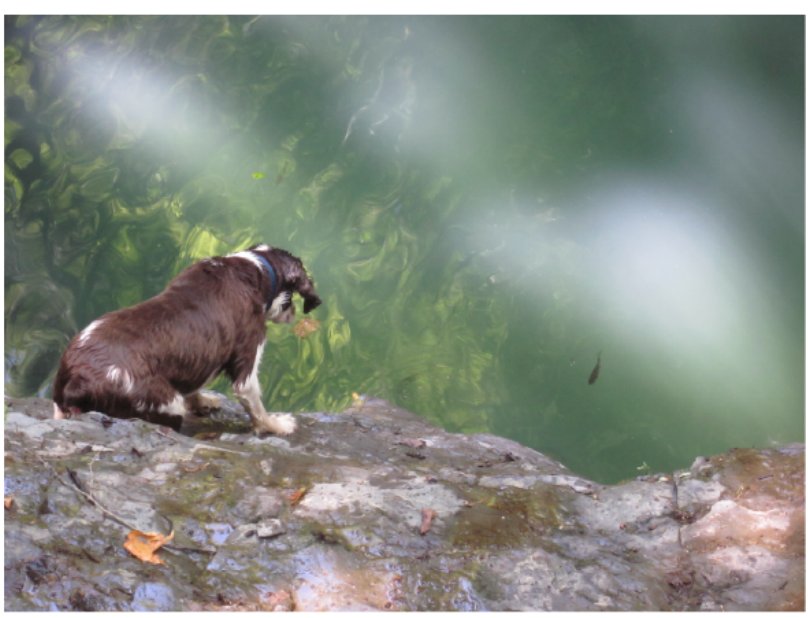

Figure 1. The Allais paradox (left) is a canonical example of what we call a discrete decision. It involves a single choice between two options; the choice is independent of action, context, and time. It has no influence on what options are available in the future. A dog moving along a bank preparing to jump and grab a fish (right) is a canonical example of a continuous decision.

\section{Foraging decisions are continuous decisions}

The study of naturalistic decisions is the central concern of foraging theory, an important branch of behavioral ecology (Stephens and Krebs, 1986; Stephens et al., 2008). The centrality of continuousness in foraging theory is demonstrated by its two foundational problems - patch-leaving and diet selection (Stephens and Krebs, 1986). In patch-leaving, the decision-maker chooses between leaving and staying; in diet selection, between accepting and rejecting an encountered prey item. And while both might appear at first 
139 glance to be discrete decisions, they are best understood as part of the framework of

140 continuous decisions as described above. Patch-leaving, for instance, is most properly

141 understood not as a binary stay or go decision, but as a when to go decision, the selection

142 of a moment to leave the current food patch in search of better options. Likewise, prey

143 selection is a binary decision — pursue this item or not? - taken against the backdrop of

144 asynchronous prey encounters. That is, both require a fuller picture of dynamic context and

145 feedback than that typically used in laboratory studies of discrete decisions. (Stephens and

146 Anderson, 2001; Blanchard et al., 2013; Pearson et al., 2010).

147 Similar arguments can be advanced for other, less well-known types of foraging

148 decisions. For instance, the tradeoffs involved in behavioral scheduling - when to eat,

149 when to sleep, when to mate - involve more than isolated value comparisons. Since each

150 of these behaviors is necessary but they are (usually) mutually exclusive, current

151 opportunities, physiological state, and recent history must therefore alter their respective

152 values to produce the observed behavioral dynamics. Thus, a "meta-task" perspective,

153 which fits naturally into the hierarchical framework for continuous decisions we describe

154 below, is both natural and necessary for real-world behavior.

155 The same holds true even more strongly for social decisions, where the presence of

156 other agents renders the choice space both larger and dynamic (Giraleau and Caraco,

157 2018). Indeed, behaviors such as swarming, flocking, and pack hunting integrate both

158 movements and decisions across multiple agents, producing a synthesis that seems

159 impossible to model with discrete decisions (Couzin and Krause, 2003, Camazine et al.,

160 2003; Viswanathan et al., 2011). And while traditional game theory has worked well to 
161 identify situations like sparse aggressive interactions that can be modeled as one-shot, 162 cases in which equilibrium behavior is informative, these methods do not naturally extend 163 to the short time-scale dynamics of social interactions. As we will argue below, extensions 164 like differential games can help address this concern, but these are best situated within the 165 broader perspective we have outlined here.

166 But perhaps the strongest argument for foraging decisions as continuous decisions 167 comes from psychophysics, where small changes in temporal contingency can result in 168 remarkable differences in task performance. For example, foragers that show steep - and 169 highly suboptimal - temporal discounting in standard laboratory setups tend to show much 170 better behavior in the foraging contexts (Hayden, 2016; Blanchard and Hayden, 2015 171 Cash-Padgett and Hayden, 2020), even when the delay structures of the task are highly 172 similar. One theory holds that the foraging context motivates the contemplation of the 173 problem as a continuous problem and, because this form of problem resembles the evolved 174 problem, it elicits more optimal behavior (Stephens and Anderson, 2001). Along similar 175 lines, it has been conjectured that this difference is related to learning, with the temporal 176 structure of foraging much easier to learn than the post-reward delays typically imposed in 177 intertemporal choice tasks (Pearson et al., 2010; Blanchard et al., 2013). This again argues 178 for the importance of considering continuous decisions as a framework better aligned with 179 the behaviors that brains evolved to organize. 
A

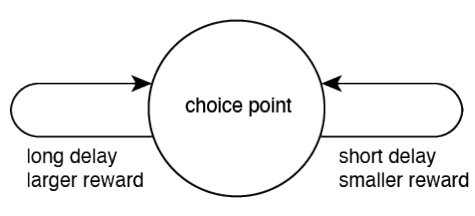

B

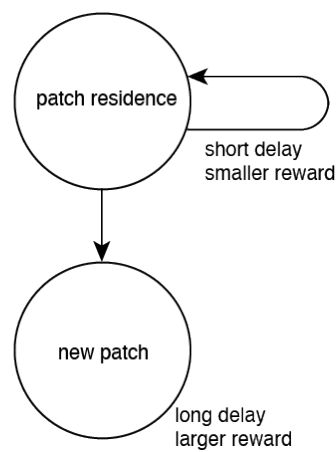

C

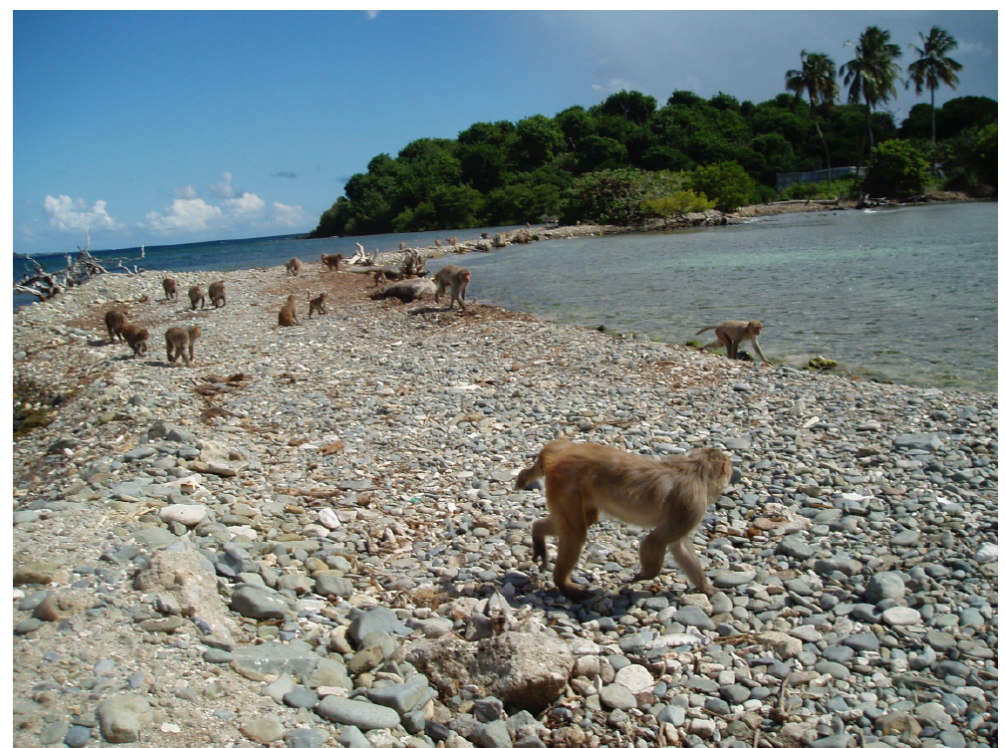

182

183

184

185

186

187

188

189

190

191

192

193

194

195

196

197

198

199

200

201

Figure 2. A. A traditional discrete choice task used to study time preferences is the intertemporal choice task (reviewed in Hayden, 2016). B. A patch-leaving task has an ostensibly isomorphic structure, except that the specific options available on the next trial are determined entirely by the choices made on the present trial (Stephens and Anderson, 2001). This feature has major effects on choice patterns and, although the element of cross-trial dependency is ostensibly more difficult, renders behavior nearly optimal (Blanchard and Hayden, 2015). C. The patch-leaving task, but not the intertemporal choice task, is ethologically valid. Here, monkeys at the Cayo Santiago field station travel between their morning foraging site on Large Cay to their afternoon foraging site on Small Cay.

\section{Continuous decisions are open-world, and the world is large}

Above, we have argued that foraging decisions, even when reduced to laboratory

tasks, point in the direction of key aspects of real-world decisions that better align with

natural behavior. Here, we will consider a related aspect of continuous decisions that sets

them apart from discrete decisions: what might be called their "open world"-ness.

We take the term "open world" from the domain of video games. Open world games like Grand Theft Auto and Minecraft present players with virtually limitless territory to explore, and terrain filled with a multitude of possible actions. Like the 
202 situations in which many real world decisions occur, they contain more unknowns than

203 knowns, which reward curiosity and search, and a large number of other agents with their

204 own goals. Open world environments pose a significant computational challenge, elided in 205 nearly all laboratory decisions, but central to real decisions, of narrowing down a plenitude 206 of possible actions to a small set of "live" options. To meet these challenges, decision207 makers must employ cognitive abilities that make these computationally intensive 208 problems tractable, abilities like mental maps, curiosity, and heuristics.

209 To sharpen this theoretically, we adopt the language of reinforcement learning 210 (RL), which offers several advantages (Sutton and Barto, 2018; Dayan and Abbott, 2001):

211 First, RL does not need to either assume a model of the world, a fixed set of choices, or 212 even a static set of values. A learner that follows RL can learn online (it does not need to 213 be pre-trained). Second, while RL has traditionally been most effective in the tabular 214 setting - the case in which options and contexts are discrete and finite, so that the value of 215 each action can be stored in a lookup table - its formalism is generic enough to extend to 216 the continuous decisions we envision here. Finally, recent progress in deep reinforcement 217 learning has opened a new dialogue between machine learning and cognitive neuroscience, 218 producing artificial agents capable of solving challenging continuous tasks like video 219 games while underperforming humans in others like imitation learning (Mnih et el., 2013;

220 Vinyals et al., 2019; Finn et al., 2017; Duan et al., 2017; Ho and Ermon, 2016).

221 In this language, the first distinction we want to draw between discrete and 222 continuous tasks is in the dimension of what is called the state space. For a given problem, 223 RL always assumes a state, the collection of variables needed to fully define the current 
224 context (Sutton and Barto, 2018; Wikenheiser and Schoenbaum, 2016; Schuck et al., 2016;

225 Wilson et al., 2014). For discrete choices, the state space (the set of all possible states)

226 usually consists of a small number of variables, perhaps attribute information for each of

227 the choice options available and some information about current preferences. For the

228 dragonfly pursuit described above, the current state might include position and velocity

229 information for all joints, estimated position and velocity of both the dragonfly and its

230 prey, and the relative locations of relevant obstacles. In addition, the state might also

231 include internal variables of the organisms that might be relevant to predicting behavior

232 (e.g. hunger, fatigue, etc). Consequently, the state space in open-world decisions tends to

233 be enormous. In part, the proliferation of these states is related to the mathematical

234 requirements of RL, which assume that the state contains all information on which

235 behavior at the current time depends. But this explosion of states is also inherent in the

236 complexity of the behavior itself and the granularity at which it is modeled.

237 Moreover, it is not just the number of state variables that are increased in

238 continuous decisions, but their ranges. In discrete RL settings, for S state variables with $\mathrm{N}$

239 values each, the size of the state space is $\mathrm{N}^{\wedge} \mathrm{S}$. That is, for a decision problem with five

240 yes/no contextual variables (Am I hungry? For sweet or salty snacks? Is there food close

241 by?), there are a total of $2^{\wedge} 5=32$ possible states. . In the case of continuous states, where $\mathrm{N}$

242 becomes infinite, state spaces become so large that tabular approaches must fail. Moreover,

243 in many cases of interest, states are only partially observed, requiring some form of state

244 inference. For example, in games like poker, opponents' cards are key missing pieces of

245 game state information. Finally, in continuous decisions, where possible actions include 
246 continuous variables like forces and joint angles, the resulting space is also infinite

247 dimensional.

248 Fortunately, while practical and algorithmic challenges remain in these cases, they

249 are addressed by existing concepts like models, maps, trajectories, and policies that bridge

250 the discrete and continuous cases. The common insight underlying these extensions of

251 tabular RL is that, when states or actions are continuous, we can often make use of a

252 concept of distance, such that "nearby" states are similar, and quantities like value can be

253 assumed to change smoothly. That is, continuous spaces can often, paradoxically, have

254 fewer variables than discrete ones. For instance, if one is given a table of 100 numbers, this

255 data structure has, apparently, 100 free parameters. But if these numbers are samples from

256 a quadratic function at regularly spaced intervals along the $\mathrm{x}$ axis, they are encapsulated by

257 only three parameters. These sorts of geometric and smoothness assumptions often allow

258 one to replace tables with functions and an infinite number of individualized values with a

259 finite number of function parameters, rendering learning feasible once again.

260 Along related lines, much recent work on reinforcement learning has focused on

261 the existence and utility of mental models or maps (Schuck et al., 2016; Behrens et al.,

26220181 Niv, 2019; Momennejad et al., 2019; Stachenfeld et al., 2017). What is important in

263 these cases is that not that such maps are necessary for learning — RL agents who lack

264 them can still eventually learn — but that these maps capture either regularities in state

265 sequences (like random walks on an unseen graph) or structure within the states (like

266 proportions of bird beaks and legs) that facilitate faster learning. In the language of

267 learning theory, they constitute inductive biases that allow learners to make the most of 
268 each observation. Similarly, in a simulated pursuit task, we found evidence for such maps

269 in the firing rates of single neurons in the dorsal anterior cingulate cortex (dACC).

270 Specifically, dACC neurons have complex, non-regular multimodal firing rate place fields

271 (Yoo et al., 2020), reminiscent of non-grid cells in the medial entorhinal cortex (Hardcastle

272 et al., 2017). More importantly, such inductive biases, by making strong assumptions about

273 the possible types of knowledge to be acquired, combat the curse of dimensionality. In the

274 studies above, though numbers of states were large, their dimensionalities remained small.

275 When dimensionality likewise increases, learning becomes an intractable search problem

276 without some form of organizing assumption.

277 By the same logic, open-world decisions necessitate exploration, since large state

278 spaces cannot be exhaustively visited. This means that continuous decision-makers are

279 almost always highly information-starved. In typical studies of discrete decisions, it is

280 assumed that decision-makers have access to all relevant information and that they have no

281 cost to process it. When information is lacking, only one form of information is excluded,

282 and its absence is typically made the focus of study. So, while discrete decisions can also

283 be information-starved, that is not the standard case. Thus, the demand for information is

284 typically a major influence on continuous decisions, whereas it is only a major influence

285 on discrete ones when they are designed that way. This is likely one reason why humans

286 and other foragers appear to have deeply evolved drives towards curiosity - willingness to

287 pay for even ostensibly non-strategic information (Kidd and Hayden, 2016; Gottlieb et al.,

288 2013; Wang and Hayden, 2019). They are trying to fill in the large gaps in their maps of

289 the world as much as possible. Indeed, much recent work in RL is focused on efficient 
290 methods of exploration in the absence of rewards - the search for an algorithmic

291 curiosity. And such a focus is relevant in worlds like ours where decision-making is more

292 often a problem of search for acceptable options than of choice between competing ones.

293 Finally, it is important to note that the goal of action is not necessarily to find the

294 optimal course of action, but instead to find a course of action that is sufficiently good

295 (Griffiths et al., 2015; Schwartz et al., 2002; Gigerenzer and Gaissmaier, 2011). Such

296 approaches are valuable even in simple non-continuous decisions (e.g. Venkatraman et al.,

297 2009; Heilbronner and Hayden, 2016). The additional complexity of natural decisions

298 means that satisficing approaches, such as heuristics, are likely to be used in real-world

299 decision-making. In continuous decisions, where the costs of computation are much greater

300 and the time pressures more unrelenting, heuristics are likely to be even more important.

301 Understanding the use of heuristics in continuous decisions, then, represents an important

302 future direction for study.

303 

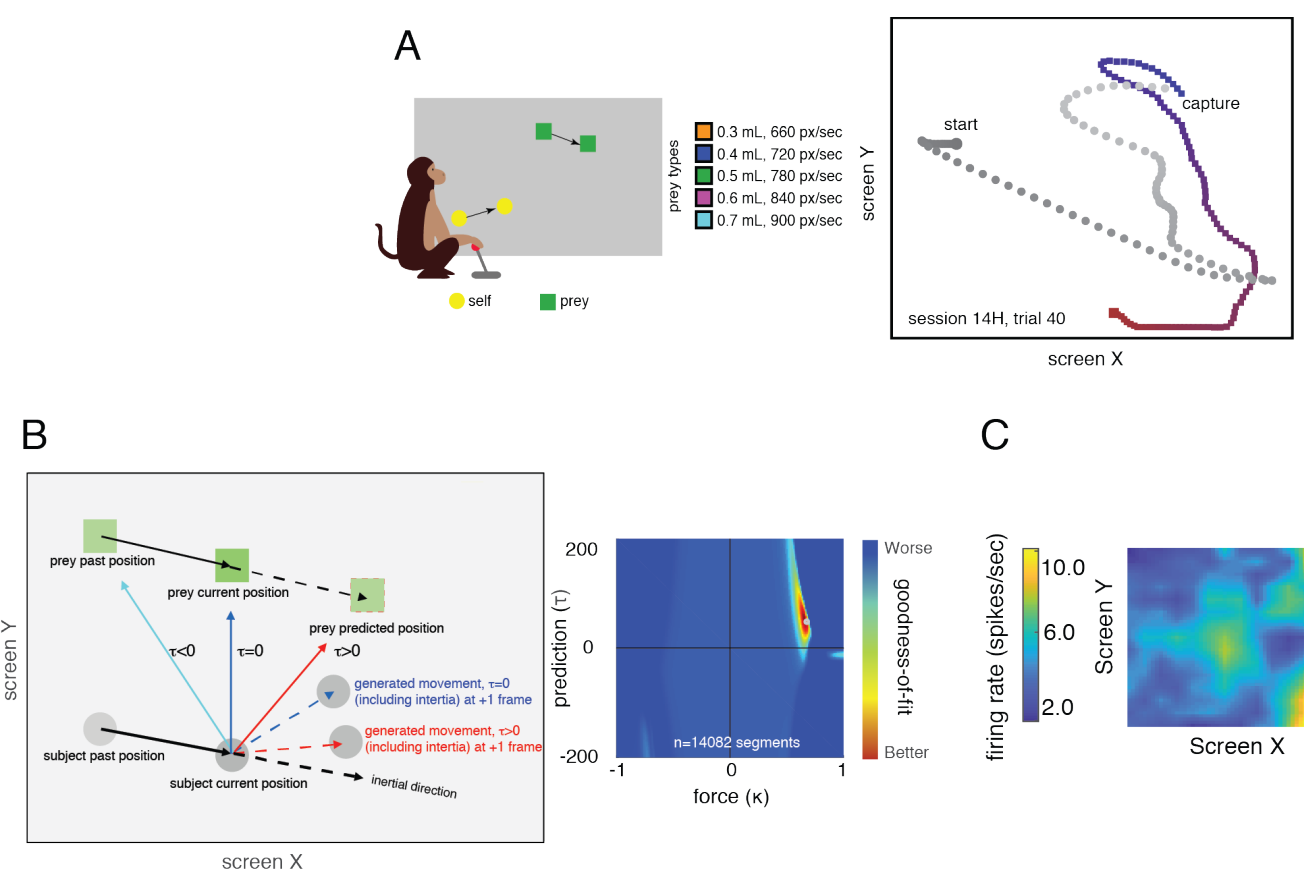

C

Figure 3. Task and data from Yoo et al., 2020. (A) (Left) Cartoon of virtual pursuit task. Subject uses a joystick to control an avatar (circle) and pursue prey (square) on a computer screen. (Right) Avatar and prey trajectories on example trials. Grey: path of avatar; red/blue: path of prey. Color gradient indicates the time progression through the trial. (B) (Left) The generative model to explain the trajectory of nonhuman primates. (Right) The model result exhibiting that nonhuman primates are predicting the future position of the prey. (C) Example filters from neurons that are significantly tuned for the position of the prey within the monitor.

\section{Continuous decisions involve dynamics and feedback}

Just as large numbers and dimensions of states advantages agents who are able to make use of abstractions like maps and world models, so tasks that involve actions

throughout time give rise to considerations of dynamics. Indeed, the importance of both dynamics (sometimes high-dimensional, often nonlinear) and feedback (from both other agents and the environment) is the most obvious feature setting off what we have called continuous decisions from their more conventional discrete counterparts. The problems imposed by dynamics encompass questions of control - how to move an arm to snatch a passing prey — and questions of timing — precisely when to make this move, adding 
323 additional complexities often excluded in laboratory decision paradigms. Once again, we

324 will argue that reinforcement learning, particularly its intersection with control theory,

325 offers a conceptual framework for addressing these challenges, even in cases where

326 learning is not the primary dynamic.

327 Before stepping further, it is helpful to draw the distinction between modeling a set

328 of actions as sequential decisions and the cognitive experience of sequential decision

329 making. For instance, while it is intuitive that the iterated Prisoner's Dilemma involves a

330 sequence of cooperation and defection decisions by each player, it is less intuitive that a

331 short walk down a straight hallway also comprises a series of decisions, one for each step.

332 Yet both can be modeled mathematically as probabilistic sequences of "decisions,"

333 perhaps, in the case of walking, with rewards for staying upright and forward progress. Yet

334 our subjective experience is one of long periods of automatic behavior punctuated by

335 changes of mind, phenomena that are only imperfectly captured by the framework of

336 sequential decisions. Instead, we might prefer to mirror mathematically the dissociation

337 between rare goal changes and continuous execution of unconscious behaviors in time.

338 The first ingredient in such a recipe comes from control theory, which has a long

339 and fruitful history in motor neuroscience (e.g. Todorov and Jordan, 2002; Wolpert et al.,

340 2011; Gallivan et al., 2018; Diedreichsen et al., 2010). In the control framework, a system

341 is endowed with a set of possible controls to apply (forces, torques, electronic commands),

342 a system to which these controls may be applied (typically modeled as a set of differential

343 equations), a goal or set point, and a cost for applying control (energy, price, regret). The

344 aim of control theory is to solve for a policy, an algorithm that sets control as a function of 
345 the system's state at a given time. The optimal policy is one that best balances control error

346 (how close the system remains to its goal) against control costs. Such a framework

347 automatically addresses two key problems identified above: First, control systems operate

348 in time. Control policies are functions, not discrete actions, and specify how the controller

349 co-evolves with the system. They are thus fundamentally dynamic quantities. Second,

350 control policies do not require the semantics of decisions at all. They are conceptualized as

351 automatic processes that operate adaptively, often deterministically, once a goal has been

352 set. Thus, they have intuitive appeal for a framework that combines sparse, conscious

353 decisions with automatic ongoing processes.

354 Again, this is complementary to the reinforcement learning perspective reviewed

355 above (RL; Sutton and Barto, 2018), which likewise attempts to learn a policy that

356 maximizes some set of rewards. In fact, many classic RL problems like cart-pole or

357 learning to walk are control problems (Todorov and Jordan, 2002; Todorov, 2004;

358 Todorov, 2009). Such links have often been overlooked in decision neuroscience, where

359 RL actions are most frequently associated with decisions (Daw and Tobler, 2014), but

360 there is nothing in the formalism to force this view. Before the advent of deep

361 reinforcement learning, RL methods were often limited to low-dimensional, discrete state

362 and action spaces, but modern applications now routinely feature complex, continuous

363 policies parameterized by deep neural networks (Mnih et al., 2015; Van Hasselt et al.,

364 2016;). Thus, RL likewise offers up the notion of a full policy in place of a discrete action 365 as a building block of continuous decisions. 
Just as importantly, while control systems are often assumed to be autonomous,

367 with fixed, pre-specified set points, RL can be extended to the case of hierarchical learning 368 and control (Doya et al., 2002; Precup \& Sutton, 2000; Pearson \& Platt, 2013; Merel et al., 369 2019; Botvinick et al., 2009). That is, the RL framework can easily accommodate the idea

370 of switching between policies or changing goals within the same policy (McDonald et al.,

371 2019; Iqbal et al., 2019). These high-level changes are most often slower (in the case of

372 goals) or sparser (for policy switches) and map neatly onto the experience of rarer

373 deliberative decisions setting in motion automatic behaviors. Moreover, hierarchical

374 formulations of RL allow changes in state to drive high-level actions, facilitating "bottom-

375 up" feedback that renders policies more flexible than purely "fire and forget" processes.

376 And while training such hierarchical RL systems has continued to prove challenging

377 (Precup and Sutton, 1998; Nachum et al., 2019), there is ample evidence that brains have

378 evolved by solving exactly this problem (Niv, 2019).

379 Two recent paradigms from our groups illustrate precisely this sort of integration of 380 continuous control with sparse strategy changes. In the first, monkeys and humans played a 381 competitive video game against conspecifics that required continuous joystick input

382 (McDonald et al., 2019; Iqbal et al., 2019, Figure 4). Based on the idea of a penalty shot in 383 hockey, one player (the shooter) controlled the motion of a small circle (the puck), while 384 the other (the goalie) controlled the vertical motion of a bar placed at the opposite side of 385 the screen. Goalies were rewarded for blocking the puck with the bar, while shooters 386 received rewards for reaching a goal line behind the bar. Though the game was highly 
387 constrained, players' trajectories proved highly variable, revealing a variety of both 388 individual strategies and inter-player dynamics.

389 These phenomena can be modeled as the result of a control model applied to an 390 evolving value function incorporating each player's trajectory (Iqbal et al., 2019;

391 McDonald et al., 2020), while directly modeling players' policies allows for the calculation 392 of a measure of instantaneous coupling between the players (McDonald et al., 2019).

393 However, in both human and monkey participants, it was found that the key strategic 394 variable was not so much shooters' ability to adjust to their opponents as their ability to 395 advantageously time their final movements on each trial (McDonald et al., 2019;). Such 396 paradigms as this bear some relation to continuous games such as duels and are closely 397 related to pursuit games in differential game theory, but in the latter, focus is typically on 398 computing optimal solutions rather than modeling players' real behavior (Basar and 399 Olsder, 1999; Braun et al., 2009). 
a

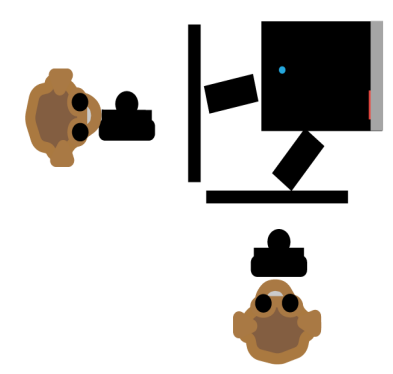

C

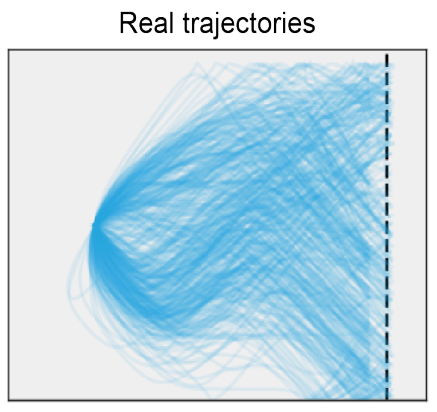

e

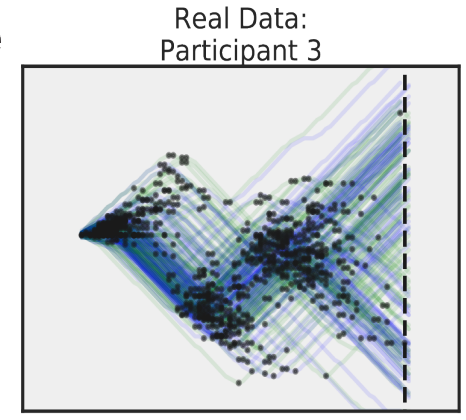

b

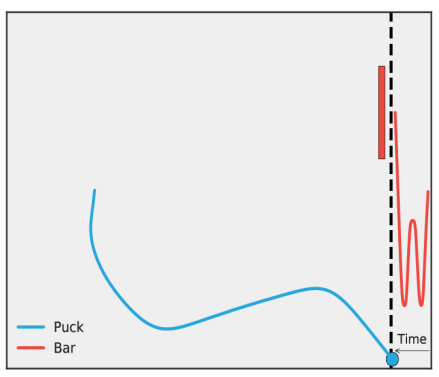

d

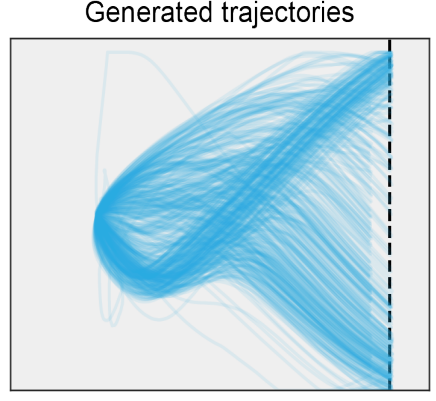

f

Model Prediction:

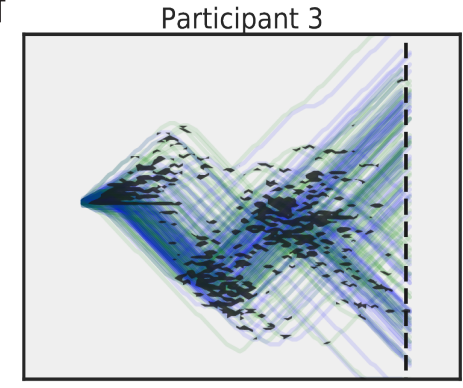

Figure 4. Task from lqbal et al., 2019. (A) Pairs of monkeys competed in a zerosum, real-time game with continuous joystick inputs. One player controlled the position of a blue ball, the other the vertical position of a red bar. The goal of the blue player was to guide the ball past the dotted line at the right of the screen, while the red player attempted to block the ball with the bar. (B) Example of a single pair of player trajectories on a single trial. For display purposes, the trajectory of the bar has been extended in time along the $x$ axis, though the bar could only move vertically. (C) Continuous control resulted in highly varied trajectories, but these could be captured by a model of players' coevolving goal states that generated realistic trials (D). (E) In a version of the same task in human subjects, players' trajectories were characterized by sparse changes in trajectory (black dots), and these changepoints could be predicted by modeling players' policies as a function of continuous game state (F). Black patches represent regions of increased probability of direction change. Blue traces indicate trials played against a human bar 
414

415

416

417

418

419

420

421

422

423

424

425

426

427

428

429

430

431

432

433

434

opponent, green traces those played against a computer. Adapted from lqbal, Yin et al., 2019; McDonald et al., 2019.

\section{Implications for neuroscience: a preliminary sketch}

As neuroscientists, our central concerns are on the neural mechanisms that drive choices. Our central argument here is for the importance of continuous decisions; our motivator is the overall lack of consideration for them in the field. As such, it may be too soon to draw any conclusions on the likely implications for neuroscience. We therefore choose to draw a narrow focus and consider one major question, that of modularity vs. distributedness.

Traditional "box-and-line" approaches to cognitive neuroscience presume the existence of discrete cognitive functions with intuitive roles. These functions, then, are assumed to be reified in neuroanatomy. For example, if choice consists of evaluation, comparison, and selection, then these three conceptually discrete functions ought to correspond to discrete anatomical substrates (Rangel et al., 2008; Padoa-Schioppa, 2011). An alternative viewpoint is distributed; it imagines that choice reflects an emergent process arising from multiple brain regions whose functions may not correspond to nameable processes, and/or that may largely overlap (Yoo et al., 2018; Hunt and Hayden, 2017;

Cisek and Kalaska, 2010; Cisek, 2012).

The greatest debates about modularity occur in the prefrontal cortex, which can be readily divided into a few dozen areas based on cytoarchitecture; however, the functions of these areas remain difficult to definitively identify. In fact, this architectural diversity raises the question of why we have so many prefrontal regions to begin with. One possibility is that each region integrates new information from new sources and 
438 participates in transforming that information via an untangling process (Yoo and Hayden,

439 2018). In this view, each area in sequence provides a partial transformation from one

440 representation format to another (Azab and Hayden, 2017 and 2018). Indeed, it is this

441 view, and not that of a modular system, that best accords with recent results in artificial

442 neural networks (DiCarlo et al., 2012). Of course, some cognitive functions are localized at

443 the coarsest levels. Our claim is simply that, once one embraces the framework of

444 continuous decisions, with its tight coupling of action and decision, dynamics and

445 feedback, the tight conceptual link between modularity in space and serial processing in

446 time is no longer so natural as it has traditionally appeared.

447 The modular assumption becomes less tenable when moving from discrete to

448 continuous decisions (Cisek, 2012). Consider choosing between two options. In this

449 stereotypical situation, the motor action may not begin until comparison and choice of

450 option are complete, and the action does not influence the decision. The serial model then

451 neglects the physicality of the decision-making agent so action is a slave of choice. There

452 are studies in which action cost is considered (refs; Hosokawa), but these are the rare

453 exceptions. In continuous decisions, action is front and center; it is difficult to even think

454 about how choice may occur without consideration of action. That includes not just action

455 costs, but also forward models of the consequences of possible strategies. In other words,

456 the obligatory embodiedness of continuous decisions means that action selection cannot be

457 separated from choice.

458 In practice, subjects appear to initiate their action before they have fully decided on 459 an option. That is, they hedge their bets and choose an intermediate path between the two 
460

461

462

463

464

465

466

467

468

469

470

471

472

473

474

475

476

477

478

479

480

481

482

options (Haith et al., 2016; Christopoulos and Schrater, 2015). Then, as their decision process unfolds in their heads, their arm changes its pattern and begins to divert its path towards the chosen option. Critically, the initial hedging path lasts longer if the choice takes more time to unfold. In other words, the beginning of selection takes place before the choice is complete. Taking this idea literally, the choice process occurs simultaneously with the motor action. They are not discrete and the action would provide a feedback signal to the choice, which is opposed to the idea that movement does not influence the choice at all. Ultimately, then, resolution of the question of modularity requires testing under more naturalistic contexts. That is, natural tasks provide a valuable "stress test" for modular models.

\section{Conclusion}

Here, we have argued in favor of a decision-making framework that generalizes

beyond discrete decisions among small, fixed option sets to continuous, open-world, dynamic processes that blend value and decision with control and movement. Of course, the greatest obstacle to the adoption of this framework, pieces of which go back decades, is the additional complexity involved in such unconstrained behaviors. As a result, we propose that the development of new experimental analysis techniques ought to be a major focus moving forward. For example, most traditional analyses in systems neuroscience have relied on averages, both in time and across trials, to reduce the complexity in data and achieve statistical power. But when trial-to-trial behavioral variability is large, these repetitions may no longer be comparable to one another. As a result, single trial analyses will be crucial (e.g. Sauerbrei et al., 2020; Pandarinath et al., 2018, also Iqbal et al., 2019; 
483 McDonald et al., 2019, 2020). And these methods often benefit from denser recording

484 techniques such as calcium imaging (Stringer et al., 2019;) and multi-contact multi-

485 electrode recording (Gallego et al., 2017, 2018; Golub et al., 2018).

Along a different axis, there is much promise in recent studies that eschew single

487 brain areas in favor of integrative analyses of simple, natural behaviors. Recent technical

488 advances are making such studies possible, and this trend is likely to continue. A final

489 example comes from problems introduced by distributed decision and control models - we

490 need theoretical approaches that explain how multiple brain regions interact constructively

491 without interfering (Hunt and Hayden, 2017; Yoo and Hayden 19; Eisenreich et al., 2017).

492 But we reiterate: to the degree that some complexity in behavior is irreducible, the

493 study of continuous decisions is both necessary and inevitable. Not only are these

494 decisions closer to natural behavior (and so easier to train in model species), they begin to

495 tap the complexity we want to study, offering new puzzles that force us to integrate our

496 knowledge across cognitive levels, brain regions, and time scales. If the behaviors we are

497 interested in--our own included--take place in a changing, dynamic world, then our models

498 of that world must grow to embrace these essential features. 
500

501

502

503

504

505

506

507

508

509

510

511

512

513

514

515

516

517

518

519

520

521

522

523

524

525

526

527

528

529

530

531

532

533

534

535

536

537

\section{REFERENCES}

Allais, M. (1953). Le comportement de l'homme rationnel devant le risque: critique des postulats et axiomes de l'école américaine. Econometrica: Journal of the Econometric Society, 503-546.

Azab, H., \& Hayden, B. Y. (2017). Correlates of decisional dynamics in the dorsal anterior cingulate cortex. PLoS biology, 15(11), e2003091.

Azab, H., \& Hayden, B. Y. (2018). Correlates of economic decisions in the dorsal and subgenual anterior cingulate cortices. European Journal of Neuroscience, 47(8), 979-993.

Basar, T., \& Olsder, G. J. (1999). Dynamic noncooperative game theory (Vol. 23). Siam.

Behrens, T. E., Muller, T. H., Whittington, J. C., Mark, S., Baram, A. B., Stachenfeld, K. L., \& Kurth-Nelson, Z. (2018). What is a cognitive map? Organizing knowledge for flexible behavior. Neuron, 100(2), 490-509.

Blanchard, T. C., Pearson, J. M., \& Hayden, B. Y. (2013). Postreward delays and systematic biases in measures of animal temporal discounting. Proceedings of the National Academy of Sciences, 110(38), 15491-15496.

Blanchard, T. C., \& Hayden, B. Y. (2015). Monkeys are more patient in a foraging task than in a standard intertemporal choice task. PloS one, 10(2).

Braun, D. A., Ortega, P. A., \& Wolpert, D. M. (2009). Nash equilibria in multi-agent motor interactions. PLoS computational biology, 5(8).

Brenner, N., Bialek, W., \& Van Steveninck, R. D. R. (2000). Adaptive rescaling maximizes information transmission. Neuron, 26(3), 695-702.

Borghuis, B. G., \& Leonardo, A. (2015). The role of motion extrapolation in amphibian prey capture. Journal of Neuroscience, 35(46), 15430-15441.

Bolton, A. D., Haesemeyer, M., Jordi, J., Schaechtle, U., Saad, F. A., Mansinghka, V. K., ... \& Engert, F. (2019). Elements of a stochastic 3D prediction engine in larval zebrafish prey capture. eLife, 8 . 
538 Brown, A. E., \& De Bivort, B. (2018). Ethology as a physical science. Nature Physics, 539 14(7), 653-657.

540

541

542

543

544 Camazine, S., Deneubourg, J. L., Franks, N. R., Sneyd, J., Bonabeau, E., \& Theraula, G.

545

546

547

548

549

550

551

552

553

554

555

556

557

558

559

560

561

562

563

564

565

566

567

568

569

570

571

572

573

574 Dayan, P., \& Abbott, L. F. (2001). Theoretical neuroscience: computational and

575 mathematical modeling of neural systems.

Calhoun, A. J., \& Hayden, B. Y. (2015). The foraging brain. Current Opinion in Behavioral Sciences, 5, 24-31. (2003). Self-organization in biological systems (Vol. 7). Princeton university press.

Cash-Padgett, T., \& Hayden, B. (2020). Behavioural variability contributes to over-staying in patchy foraging. Biology Letters, 16(3), 20190915.

Charnov, E. L. (1976). Optimal foraging, the marginal value theorem.

Christopoulos, V., \& Schrater, P. R. (2015). Dynamic integration of value information into a common probability currency as a theory for flexible decision making. PLoS computational biology, 11(9).

Cisek, P. (2012). Making decisions through a distributed consensus. Current opinion in neurobiology, 22(6), 927-936.

Cisek, P., \& Kalaska, J. F. (2010). Neural mechanisms for interacting with a world full of action choices. Annual review of neuroscience, 33, 269-298.

Couzin, I. D., \& Krause, J. (2003). Self-organization and collective behavior in vertebrates. Advances in the Study of Behavior, 32(1), 10-1016.

David, S. V., Vinje, W. E., \& Gallant, J. L. (2004). Natural stimulus statistics alter the receptive field structure of v1 neurons. Journal of Neuroscience, 24(31), 6991-7006.

576 
577 DiCarlo, J. J., Zoccolan, D., \& Rust, N. C. (2012). How does the brain solve visual object

578 recognition?. Neuron, 73(3), 415-434.

579

580

581

582

583

584

585

586

587

588

589

590

591

592

593

594

595

596

597

598

599

600

601

602

603

604

605

606

607

608

609

610

611

612

613

614

615

Diedrichsen, J., Shadmehr, R., \& Ivry, R. B. (2010). The coordination of movement: optimal feedback control and beyond. Trends in cognitive sciences, 14(1), 31-39.

Doya, K., Samejima, K., Katagiri, K. I., \& Kawato, M. (2002). Multiple model-based reinforcement learning. Neural computation, 14(6), 1347-1369.

Doya, K. (2000). Reinforcement learning in continuous time and space. Neural computation, 12(1), 219-245.

Duan, Y., Andrychowicz, M., Stadie, B., Ho, O. J., Schneider, J., Sutskever, I., ... \& Zaremba, W. (2017). One-shot imitation learning. In Advances in neural information processing systems (pp. 1087-1098).

Eisenreich, B. R., Akaishi, R., \& Hayden, B. Y. (2017). Control without controllers: toward a distributed neuroscience of executive control. Journal of cognitive neuroscience, 29(10), 1684-1698.

Farashahi, S., Azab, H., Hayden, B., \& Soltani, A. (2018). On the flexibility of basic risk attitudes in monkeys. Journal of Neuroscience, 38(18), 4383-4398.

Finn, C., Yu, T., Zhang, T., Abbeel, P., \& Levine, S. (2017). One-shot visual imitation learning via meta-learning. arXiv preprint arXiv:1709.04905.

Fusi, S., Miller, E. K., \& Rigotti, M. (2016). Why neurons mix: high dimensionality for higher cognition. Current opinion in neurobiology, 37, 66-74.

Gallego, J.A., Perich, M.G., Miller, L.E., \& Solla. S.A. (2017) Neural Manifolds for the Control of Movement. Neuron. 94, 978-984.

Gallego, J.A., Perich, M.G., Chowdhury, R.H., Solla. S.A., \& Miller, L.E. (2020) Longterm stability of cortical population dynamics underlying consistent behavior. Nature Neuroscience, 23, 260-270.

Gallivan, J. P., Chapman, C. S., Wolpert, D. M., \& Flanagan, J. R. (2018). Decisionmaking in sensorimotor control. Nature Reviews Neuroscience, 19(9), 519-534. 
616 Gershman, S. J. (2018). The successor representation: its computational logic and neural

617 substrates. Journal of Neuroscience, 38(33), 7193-7200.

618

619 Gigerenzer, G., \& Gaissmaier, W. (2011). Heuristic decision making. Annual review of 620 psychology, 62, 451-482.

621

622

Giraldeau, L. A., \& Caraco, T. (2018). Social foraging theory (Vol. 73). Princeton

623 University Press.

624

625 Glimcher, P. W., \& Fehr, E. (Eds.). (2013). Neuroeconomics: Decision making and the 626 brain. Academic Press.

627

628 Gonzalez-Bellido, P. T., Fabian, S. T., \& Nordström, K. (2016). Target detection in 629 insects: optical, neural and behavioral optimizations. Current opinion in neurobiology, 41, 630 122-128.

631

632 Gottlieb, J., Oudeyer, P. Y., Lopes, M., \& Baranes, A. (2013). Information-seeking, 633 curiosity, and attention: computational and neural mechanisms. Trends in cognitive 634 sciences, 17(11), 585-593.

635

636

Griffiths, T. L., Lieder, F., \& Goodman, N. D. (2015). Rational use of cognitive resources:

637 Levels of analysis between the computational and the algorithmic. Topics in cognitive

638 science, 7(2), 217-229.

639

640 Haith, A. M., Pakpoor, J., \& Krakauer, J. W. (2016). Independence of movement

641 preparation and movement initiation. Journal of Neuroscience, 36(10), 3007-3015.

642

643 Hardcastle, K., Maheswaranathan, N., Ganguli, S., \& Giocomo, L. M. (2017). A

644 multiplexed, heterogeneous, and adaptive code for navigation in medial entorhinal cortex.

645 Neuron, 94(2), 375-387.

646

647 Hayden, B. Y. (2016). Time discounting and time preference in animals: a critical review.

648 Psychonomic bulletin \& review, 23(1), 39-53.

649

650 Heilbronner, S. R., \& Hayden, B. Y. (2016). The description-experience gap in risky

651 choice in nonhuman primates. Psychonomic bulletin \& review, 23(2), 593-600.

652

653 Ho, J., \& Ermon, S. (2016). Generative adversarial imitation learning. In Advances in

654 neural information processing systems (pp. 4565-4573). 
655

656

657

658

659

660

661

662

663

664

665

666

667

668

669

670

671

672

673

674

675

676

677

678

679

680

681

682

683

684

685

686

687

688

689

690

691

692

693

Hunt, L. T., \& Hayden, B. Y. (2017). A distributed, hierarchical and recurrent framework for reward-based choice. Nature Reviews Neuroscience, 18(3), 172-182.

Iqbal, S. N., Yin, L., Drucker, C. B., Kuang, Q., Gariépy, J. F., Platt, M. L., \& Pearson, J. M. (2019). Latent goal models for dynamic strategic interaction. PLoS computational biology, 15(3), e1006895.

Kacelnik, A., Vasconcelos, M., Monteiro, T., \& Aw, J. (2011). Darwin's “tug-of-war” vs. starlings" "horse-racing": how adaptations for sequential encounters drive simultaneous choice. Behavioral Ecology and Sociobiology, 65(3), 547-558.

Kahneman D, Tversky A. 1979 Prospect theory: an 9. analysis of decision under risk. Econometrica 47, 263 - 292. (doi:10.2307/1914185)

Kidd, C., \& Hayden, B. Y. (2015). The psychology and neuroscience of curiosity. Neuron, 88(3), 449-460.

Kobak, D., Brendel, W., Constantinidis, C., Feierstein, C. E., Kepecs, A., Mainen, Z. F., ... \& Machens, C. K. (2016). Demixed principal component analysis of neural population data. Elife, 5, e10989.

Krakauer, J. W., Ghazanfar, A. A., Gomez-Marin, A., MacIver, M. A., \& Poeppel, D. (2017). Neuroscience needs behavior: correcting a reductionist bias. Neuron, 93(3), 480490.

Lepora, N. F., \& Pezzulo, G. (2015). Embodied choice: how action influences perceptual decision making. PLoS computational biology, 11(4).

Mante, V., Sussillo, D., Shenoy, K. V., \& Newsome, W. T. (2013). Context-dependent computation by recurrent dynamics in prefrontal cortex. nature, 503(7474), 78-84.

McDonald, K. R., Broderick, W. F., Huettel, S. A., \& Pearson, J. M. (2019). Bayesian nonparametric models characterize instantaneous strategies in a competitive dynamic game. Nature communications, 10(1), 1-12.

McDonald, K. R., Pearson, J. M., and Huettel, S. A. (2020). Dorsolateral and dorsomedial prefrontal cortex track distinct properties of dynamic social behavior. Social, Cognitive, and Affective Neuroscience 
694

695

696

697

698

699

700

701

702

703

704

705

706

707

708

709

710

711

712

713

714

715

716

717

718

719

720

721

722

723

724

725

726

727

728

729

730

731

732

Merel, J., Botvinick, M., \& Wayne, G. (2019). Hierarchical motor control in mammals and machines. Nature Communications, 10(1), 1-12.

Mnih, V., Kavukcuoglu, K., Silver, D., Graves, A., Antonoglou, I., Wierstra, D., \&

Riedmiller, M. (2013). Playing atari with deep reinforcement learning. arXiv preprint arXiv:1312.5602.

Mnih, V., Kavukcuoglu, K., Silver, D., Rusu, A. A., Veness, J., Bellemare, M. G., ... \& Petersen, S. (2015). Human-level control through deep reinforcement learning. Nature, 518(7540), 529-533.

Momennejad, I., Russek, E. M., Cheong, J. H., Botvinick, M. M., Daw, N. D., \& Gershman, S. J. (2017). The successor representation in human reinforcement learning. Nature Human Behaviour, 1(9), 680-692.

Nachum, O., Tang, H., Lu, X., Gu, S., Lee, H., \& Levine, S. (2019). Why Does Hierarchy (Sometimes) Work So Well in Reinforcement Learning?. arXiv preprint arXiv:1909.10618.

Niv, Y. (2019). Learning task-state representations. Nature neuroscience, 22(10), 15441553.

Padoa-Schioppa, C. (2011). Neurobiology of economic choice: a good-based model. Annual review of neuroscience, 34, 333-359.

Pandarinath, C., O’Shea, D. J., Collins, J., Jozefowicz, R., Stavisky, S. D., Kao, J. C., ... \& Henderson, J. M. (2018). Inferring single-trial neural population dynamics using sequential auto-encoders. Nature methods, 15(10), 805-815.

Pearson, J. M., Watson, K. K., \& Platt, M. L. (2014). Decision making: the neuroethological turn. Neuron, 82(5), 950-965.

Pearson, J., Hayden, B., \& Platt, M. (2010). Explicit information reduces discounting behavior in monkeys. Frontiers in psychology, 1, 237.

Pearson, J. M., \& Platt, M. L. (2013). Change detection, multiple controllers, and dynamic environments: insights from the brain. Journal of the experimental analysis of behavior, 99(1), 74-84. 
733 Pezzulo, G., \& Cisek, P. (2016). Navigating the affordance landscape: feedback control as

734 a process model of behavior and cognition. Trends in cognitive sciences, 20(6), 414-424.

735

736

737

738

739

740

741

742

743

744

745

746

747

748

749

750

751

752

753

754

755

756

757

758

759

760

761

762

763

764

765

766

767

768

769

770

771
Pirrone, A., Azab, H., Hayden, B. Y., Stafford, T., \& Marshall, J. A. (2018). Evidence for the speed-value trade-off: Human and monkey decision making is magnitude sensitive. Decision, 5(2), 129.

Platt, M. L., \& Huettel, S. A. (2008). Risky business: the neuroeconomics of decision making under uncertainty. Nature neuroscience, 11(4), 398-403.

Precup, D., \& Sutton, R. S. (1998). Multi-time models for temporally abstract planning. In Advances in neural information processing systems (pp. 1050-1056).

Rangel, A., Camerer, C., \& Montague, P. R. (2008). A framework for studying the neurobiology of value-based decision making. Nature reviews neuroscience, 9(7), 545-556.

Resulaj, A., Kiani, R., Wolpert, D. M., \& Shadlen, M. N. (2009). Changes of mind in decision-making. Nature, 461(7261), 263-266.

Rigotti, M., Barak, O., Warden, M. R., Wang, X. J., Daw, N. D., Miller, E. K., \& Fusi, S. (2013). The importance of mixed selectivity in complex cognitive tasks. Nature, 497(7451), 585-590.

Sauerbrei, B. A., Guo, J. Z., Cohen, J. D., Mischiati, M., Guo, W., Kabra, M., ... \& Hantman, A. W. (2020). Cortical pattern generation during dexterous movement is inputdriven. Nature, 577(7790), 386-391.

Schuck, N. W., Cai, M. B., Wilson, R. C., \& Niv, Y. (2016). Human orbitofrontal cortex represents a cognitive map of state space. Neuron, 91(6), 1402-1412.

Schwartz, B., Ward, A., Monterosso, J., Lyubomirsky, S., White, K., \& Lehman, D. R. (2002). Maximizing versus satisficing: Happiness is a matter of choice. Journal of personality and social psychology, 83(5), 1178.

Sleezer, B. J., Castagno, M. D., \& Hayden, B. Y. (2016). Rule encoding in orbitofrontal cortex and striatum guides selection. Journal of Neuroscience, 36(44), 11223-11237.

Stachenfeld, K. L., Botvinick, M. M., \& Gershman, S. J. (2017). The hippocampus as a predictive map. Nature neuroscience, 20(11), 1643. 
772

773

774

775

776

777

778

779

780

781

782

783

784

785

786

787

788

789

790

791

792

793

794

795

796

797

798

799

800

801

802

803

804

805

806

807

808

809

Stephens, D. W., \& Krebs, J. R. (1986). Foraging theory. Princeton University Press.

Stephens, D. W., \& Anderson, D. (2001). The adaptive value of preference for immediacy: when shortsighted rules have farsighted consequences. Behavioral Ecology, 12(3), 330339.

Stephens, D. W., Brown, J. S., \& Ydenberg, R. C. (Eds.). (2008). Foraging: behavior and ecology. University of Chicago Press.

Stokes, M. G., Kusunoki, M., Sigala, N., Nili, H., Gaffan, D., \& Duncan, J. (2013). Dynamic coding for cognitive control in prefrontal cortex. Neuron, 78(2), 364-375.

Stringer, C., Pachitariu, M., Steinmetz, N., Reddy, C. B., Carandini, M., \& Harris, K. D. (2019). Spontaneous behaviors drive multidimensional, brainwide activity. Science, 364(6437), eaav7893.

Sutton, R. S., \& Barto, A. G. (2018). Reinforcement learning: An introduction. MIT press.

Todorov, E. (2004). Optimality principles in sensorimotor control. Nature neuroscience, 7(9), 907-915.

Todorov, E. (2009). Efficient computation of optimal actions. Proceedings of the national academy of sciences, 106(28), 11478-11483.

Todorov, E., \& Jordan, M. I. (2002). Optimal feedback control as a theory of motor coordination. Nature neuroscience, 5(11), 1226-1235.

Van Hasselt, H., Guez, A., \& Silver, D. (2016, March). Deep reinforcement learning with double q-learning. In Thirtieth AAAI conference on artificial intelligence.

Venkatraman, V., Payne, J. W., Bettman, J. R., Luce, M. F., \& Huettel, S. A. (2009). Separate neural mechanisms underlie choices and strategic preferences in risky decision making. Neuron, 62(4), 593-602.

Vinje, W. E., \& Gallant, J. L. (2000). Sparse coding and decorrelation in primary visual cortex during natural vision. Science, 287(5456), 1273-1276. 
810 Vinyals, O., Babuschkin, I., Czarnecki, W. M., Mathieu, M., Dudzik, A., Chung, J., ... \&

811 Oh, J. (2019). Grandmaster level in StarCraft II using multi-agent reinforcement learning.

812 Nature, 575(7782), 350-354.

813

814 Viswanathan, G. M., Da Luz, M. G., Raposo, E. P., \& Stanley, H. E. (2011). The physics

815 of foraging: an introduction to random searches and biological encounters. Cambridge

816 University Press.

817

818 Wang, M. Z., \& Hayden, B. Y. (2019). Monkeys are curious about counterfactual

819 outcomes. Cognition, 189, 1-10.

820

821 Wilson, R. C., Takahashi, Y. K., Schoenbaum, G., \& Niv, Y. (2014). Orbitofrontal cortex 822 as a cognitive map of task space. Neuron, 81(2), 267-279.

823

824 Wikenheiser, A. M., \& Schoenbaum, G. (2016). Over the river, through the woods:

825

826 cognitive maps in the hippocampus and orbitofrontal cortex. Nature Reviews

827

828 Wolpert, D. M., Diedrichsen, J., \& Flanagan, J. R. (2011). Principles of sensorimotor

829 learning. Nature Reviews Neuroscience, 12(12), 739-751.

830

831 Yoo, S. B. M., \& Hayden, B. Y. (2018). Economic Choice as an Untangling of Options 832 into Actions. Neuron, 99, 434-447.

833

834 Yoo, S. B. M., \& Hayden, B. Y. (2020). The Transition from Evaluation to Selection

835 Involves Neural Subspace Reorganization in Core Reward Regions. Neuron, 105, 1-13.

836

837 Yoo, S. B. M., Tu, J. C., Piantadosi, S. T., \& Hayden, B. Y. (2020). The neural basis of 838 predictive pursuit. Nature Neuroscience, 1-8.

839

840 Yuste, R. (2015). From the neuron doctrine to neural networks. Nature reviews

841 neuroscience, 16(8), 487-497.

842

843

844 